\title{
CONGRÈS DE L'AMÉNAGEMENT HYDRAULIQUE du Sud-Ouest
}

\section{L'Électrification des Chemins de Fer du Midi.}

\author{
Par M. BACHELLERY, Ingénieur en chéf du malériel et de la traction de la Compagnie des Chemins de fer du Midi.
}

C'est en 1902 que se posa pour la jremiere fois pour la Compagnie des Chemins de fer du Midi le problème de la traction élec. trique, à l'occasion de la concession de la ligne à voie étroite de Villefranche-Vernel-Ies-13ains à Boirog-Madame.

Cette ligne, établie dans une région des plus montagneuses, se présentait dans des condilions de tracé el de prolil assez comparables à celles de la ligne du Fayet à Chamonix construile quelques années auparavant et où l'exploilation électrique avait donné toute salislaction.

En conséquence, la Compagnie du Midi se décida en faveur d'une solution analogue.

Elle fit choix du système à courant continu et à troisième rail ct adopla pour la lension sur le rail 850 volts, chiffre qui, à cette époque, n'avait pás encore été atteint en Europe pour un conducteur de ce genre.

La ligne de Villefranche à Bourg-Madame fut mise en service en 1910 et, depuis celle dale, l'exploitation électrique y fonctionne dans d'excellentes conditions el: avec une régularité parfaite.

Entre lemps la question avail fait un nouveau pas avec la concession en 1907 des deux lignes transpyrénéennes d'Ax-lesThermes à Puigcerda et d'Oloron à Canfranc, lignes dont la construction, envisagee depuis fort longtemps, avait toujours été différée en raison de l'énormité des dépenses qu'elle cnlrainat. Il paraissait varaisemblable que la traction électrique, en donnant le moyen d'adopter des rayons de courbes plus réduits et des déclivités plus fortes que la traction à vapeur, permettrait d'abaisser notablement le coût de celte construclion. La convention de concession lut donc établie dans les deux hypothèses de la traction à vapeur et de la traction électrique et c'est l'étude complète des conditions d'établissement, de ces lignes qui fit définitivement pencher la balance en faveur de cetle dernière solution.

On pouvail dès lors se demander si, mème sur les lignes à voie normale déjà en exploitation dans la région des Pyrénées et dont cortaines présenlent un profil très diflicile, la traction électrique ne pourrait pas ctre avantageusement substituée à la traction à vapeur grâce à l'utilisation des forces hydrauliques disponibles dans cette région.

C'est afin d'élucider ce problème qu'à partir de 1911, la Compagnie du Midi se livra à d'importantes recherches sur la traction électrique appliquée aux grandes lignes.

Les expériences portèrent cette fois sur l'emploi de courant monophasé à haute tension capté sur un fil aérien, système qui comportait quelques applications assez étendues aux Etats-Unis el en Allemagne, et ne devait pas tarder à remporter en Suisse un incontestable succes avec l'ouverture de la ligne du Loetschberg.

Les caractéristiques de courant choisies furent la tension de 12.000 volts déjà sanctionnée par la pratique el la fréquence de 16 périodes par seconde.

Le type d'équipoment de ligne adopté fut le système dit catenaire, dont l'expérience de certains réseaux étrangers avait dès cette époque démontré les avantages.
Les nouveaux essais eurenl lieu sur la section d'llle à Villefranche dans les Pyrénées-OrienLales. Sur ce parcours de 24 kilomètres on installa, en vue d'étudier les meilleures conditions d'établissement des lignes calénaires, six types dillérents d'équipements ; on procéda à des essais complets sur six locomotives présentées par divers constructeurs suivant un programme commun tracé par la Compagnie.

En présence des résultats favorables obtenus, la Compagnie décidait de procéder immédiatement à l'électrification, d'une part de la ligne de Perpignan à Villefranche (47. kilomètres), afin d'utiliser les installations déjà réalisées, et d'autre part de la section de Pau à Montrejeau avec ses embranchements de Picrrefitle, Bagnères, Arreau et Luchon représentant 215 kilomètres de lignes dont une grande partic à fort trafic et sur lesquelles les déclivités alteignent par endroits jusqu'à 32 millimètres par mètre.

L'équipement électrique de ces différentes lignes fut entrepris aussitôt : 30 automotrices et 24 locomolives à courant moncphasé furcnt commandées en vue de leur exploitation.

Deux usines génératrices, l'une de 4.400 chevaux à Fonlpedrouse (Pyrénées-Orientales), l'autre de 21.000 chevaux à Soulom dans les Pyrénées Centrales furent construites pour leur fournir l'énergie nécessaire.

Cependant, au cours des essais, s'étail révélé un inconvénient sérieux de la traction à courant monophasé. Les lignes télégraphiques et téléphoniques disposées le long de la voie subissaient l'influence du courant de traclion et il en résultait dans les communications des troubles fort gênants.

La Compagnie du Midi dut donc s'altaquer d'urgence à ce nouveau problème extrêmement ardu, et ce n'est qu'à la veille mème de la guerre que ses recherches aboulissaient enfin à une solution acceptable consistant en l'emploi d'un fil de contre-tension et d'un feeder de retour avec transformateurs succurs, grâce auxquels les effets d'induction électrostalique et électromagnétique peuvent être pratiquement annulés.

La guerre, en absorbant en vue des nécessités de la défense nationale toutes les ressources et tous les moyens des réseaux français, vint mettre provisoirement un terme aux travaux d'électrilication des Chemins de fer du Midi.

Tout au moins, en précence de la pénurie et de l'enchérissement du charbon qui ne tardait pas à se manifester, la Compagnie voulut-elle utiliser dans toute la mesure possible les installations et le matériel déjà construits, en mettant en service la traction électrique d'une part entre Perpignan et Villefranche, d'autre part sur les embranchements de Pierrefitte, Bagnères et Arreau. L'utilisation sur ces lignes, pendant toute la guerre, de la houille blanche sous forme d'énergic électrique pour la traction permit de réaliser des économies très importantes, tout en épargnant aux populations de ces vallées privilégices une partie des restrictions de trafic que les circonstances imposaient partout ailleurs.

En même tcmps, les importantes disponibilités d'énergie des usines de la Compagnie du Midi étaient mises à la disposition d'industrics travaillant pour la défense nationale et contribuaient à la fabrication du matériel de guerre et des munitions. 
Dans ce même but et sur la demande des autorités militaires, une nouvelle usine, celle d'Eget dans la vallée d'Aure, d'une puissance de 35.000 chevaux, dont la construction avait été décidée antérieurement, était entreprise en pleine guerre. Son achèvement toutefois n'eut lieu que postérieurement à la lin des hostilités.

La guerre terminée, la Compagnie se préoccupa de reprendre la réalisation de ses projets et, les expériences précédentes lui paraissant concluantes, elle envisagea même une extension considérable de son programme, devant aboutir à l'électrification d'une fraction importante du réseau, ct amener la locomotive électrique jusqu'à Bordeaux, Toulouse, Narbonne, Cerbère et Hendaye.

Toutefois, sous l'influence des événements, une évolution s'était produite en France dans la manière de concevoir la solidarité nécessaire entre les différents éléments de l'aclivité nationale.

A l'individualisme parfois excossif dont la guerre avait révélé certains inconvénients, on cherchait à substituer, tant dans le domaine des chemins de fer que dans celui des distributions d'énergie électricque, la notion du travail en commun et de l'unification des méthodes.

La conséquence pratique en fut, en ce qui concerne l'électrification des chemins de fer, la constitution au Ministère des Travaux publics, dès l'automne de 1918, d'une Commission chargée de rechercher un système unique de traction électrique qui pût être accepté par tous les réseaux français.

Les travaux de cette Commission, qui comportèrent plusieurs missions à l'étranger, furent sanctionnés par un avis du Conseil Supérieur des Travaux publics homologné par une Décision Ministérielle du 29 août 1920 .

D'une part, pour permettre la connexion des lignes de transport d'énergie électrique des différents réseaux de chemins de fer entre elles et avec celles des réseaux de distribution, la forme de courant choisie pour ce transport et, par conséquent, pour la production dans les usines génératrices, ètait le courant triphasé et la fréquence de 50 périodes par seconde.

D'autre part, afin de permettre aux locomotives électriques d'un réseau quelconque de circuler sur toutes les lignes électrifiées du territoire national, un système de traction uniforme était adopté pour toutes ces lignes : le svstème à courant continu à la tension de 1.500 volts.

Exeeptionnellement, la tension de 3.000 volts en un ou deux points était admise pour des lignes présentant des conditions particulières d'installation ou d'exploitation, à la condition que les locomotives spécialement établies pour ces lignes pussent également être utilisées sur les lignes à 1.500 volts.

La prise de courant pouvait être aérienne ou à troisième rail, étant entendu que tous les locomoteurs devraient comporter des appareils de contact leur permettant de circuler sur des lignes équipées suivant l'un ou l'autre de ces systèmes.

Cette solution commune, à laquelle la Compagnie du Midi s'était ralliée, impliquait pour elle le sacrifice presque complet des travaux d'électrification déjà réalisés sur son réseau. Usines génératrices, lignes de transport, sous-stations, équipement des lignes, matériel moteur, presque rien de ce qui avait été fait n'était plus utilisable sans de profondes transformations. Les études d'électrifications nouvelles étaient clles-mêmes à reprendre entièrement.

Sans se décourager devant l'importance de cette tâche, la Compagnie se mit résolument à l'ouvrage.

La Décision Ministérielle du 29 août 1920 avait approuvé le programme général d'électrification présenté par les réseaux, comprenant en particulier sur le Midi 2.684 kilomètres de lignes existantes et 469 kilomètres de lignes nouvelles en construction ou projetées, soit an total 3.153 lkilometres, ou envuron les trois quarts du réseau.

Forte de cette approbation, la Compagnie remit ses projets sur le chantier ; elle entreprit la transformation des installations existantes et l'équipement de nouvelles lignes en commençant par la région ouest de son réseau. Elle mit en construction les grandes lignes de transport d'énergie qui doivent relier les Pyi énées à Bordeaux et à Toulousc. Elle fit étudier et commanda des locomotives et automotrices à courant continu. $A$ près dcux ans de travail, elle est aujourd'hui à la veille de récolter le fruit de ses efforts par la mise en service des premières installations françaises de traction à courant continu à 1.500 volts et des premières lignes françaises de transport d'énergie à 150.000 volts.

Dès cette année, on verra des trains remorqués électriquement entre Pau et Montrejeau et le courant monophasé fera place au courant continu sur les embranchements des Pyrénées Centrales. En 1923 l'exploitation électrique sera poussée d'un côté jusqu'à Toulouse, de l'autre jusqu'à Dax pour attcindre ultérieurement Bordeaux, Bayonne et Hendaye.

Cette première phase sera complétée par l'électrificalion de divers embranchements tels que ceux d'Arcachon, Biarritz, Saint-Jean-Pied-de-Port et des lignes de Morcenx à Tarbes, de Tarbes à Agen et de Toulouse à Auch. Enfin, dans la même période seront vraisemblablement mises en exploitation les deux lignes transpyrénéennes d'Oloron et d'Ax-les-Thermes dont l'exploitation électrique sera prolongée pour la première jusqu'à Pau, pour la seconde jusqu'à Toulouse.

L'électrification des lignes du versant méditerranéen constituera une deuxième étape, portant sur la ligne de Narbonne à Cerbère et ses embranchements, y compris la ligne de Rivesaltes à Carcassonne par Quillan et celles de Pamiers à Limoux et de Bram à Lavelanet.

A ce moment, le courant continu sera substitué au courant monophasé sur la ligne de Perpignan à Villefranche.

Enfin, dans les Cévennes, la Compagnie du Midi envisage encore l'électrification de la ligne de Béziers à Neussargues et de ses divers embranchements.

Lorsque ce programme sera réalisé, il ne restera plus sur le réseau du Midi, comme domaine de la locomotive à vapeur, que la grande ligne de Bordeaux à Cette et un certain nombre des lignes secondaires qui s'y embranchent.

Ainsi qu'on le voit, le programme d'électrification de la Compagnie du Midi s'est considérablement élargi depuis quelques années. Limité au début à des lignes montagneuses, il s'étend aujourd'hui à des lignes de plaine à grande circulation comme celles de Bordeaux à Hendaye et de Narbonne à Cerbère.

II n'est pas inutile d'indiquer ici quels sont les motifs de cette évolution et quels avantages la Compagnic compte obtenir de l'application extensive de la traction électricue.

Sur les lignes de montagne, les avantages de ce mode de traction sont bien connus. Ils dérivent principalement de la possibilité de constituer des unités motrices d'une puissance continue très supérieure à celle des locomotives à vapeur pour un même encombrement et une même charge par essieu.

D'une part, en effet l'effort à la jante de chaque essieu peut être plus grand en raison de ce que la locomotive électrique utilise mieux son adhérence que la locomotive à vapeur.

D'autre part, la construction articulée et le contrôle à unités multiples permettent aisément de multiplicr les essicux moteurs dans un même train. En outre, la suppression du tender et des approvisionnements élimine un poids mort important et augmente d'autant la puissance utile disponible.

Enfin, le locomoteur électrique, affranchi de toute sujétion relative à la génération de l'énergie, ne connaît pas les limita- 
tions quí s’imposent de ce fait à la machine à vapeur à la montéc des rampes, et peut, par suite, gravir celles-ci à une vitesse beaucoup plus grande.

Comme résultat de cet accroissement de puissance, on a pu adopter pour les lignes de montagne à traction électrique des caractéristiques de profil auxquelles il n'aurait pas été possible de songer avec la traction à vapeur. Sur les lignes transpyrénéennes, les rampes ont été portées jusqu'à 43 millimètres par mètre, alors que jusqu'à présent, on ne dépassait pas pratiquement sur les chemins de fer à voie normale un maximum de 35 millimètres. Sur les lignes à voie étroite on a été plus loin encore ; celle de Villefranche à Bourg-Madame présente des déclivités de 60 millimètres ; celle du Fayet à Chamonix va jusqu'à 90 millimètres.

On a pu sur les lignes existantes où la traction électrique a été substituée à la traction à vapeur augmenter dans une forte proportion, à la montée des rampes, le poids et la vitesse des trains et reculer ainsi notablement la limite de capacité de ces lignes au point d'éviter parfois des doublements de voie extrêmement onéreux.

Cette considération peut être très importante sur des lignes qui, avec la traction à vapeur, sont arrivées à la limite de leur capacité ou qui même, comme par exemple celle de Béziers à Neussargues, sont incapables d'écouler le trafic qui leur reviendrait normalement.

D'autre part, la suppression de la fumée est un avantage précieux à la traversée des longs souterrains dont la ventilation constitue un problème souvent difficile. Rappelons à ce propos que les lignes transpyrénéennes comportent plusieurs tunnels de grande longueur, dont deux respectivement de 5.300 et 7.800 mètres.

Enfin l'emploi du freinage électrique par récupération pour la descente des trains sur les fortes pentes constitue, au point de vue de la sécurité, de la facilité de réglage de l'allure, et de l'économic de sabots de frein et de bandages, l'équivalent du frcinage à contre-vapeur pratiqué depuis fort longtemps sur les Chemins de fer du Midi ; mais il permet en outre d'utiliser sur le réseau ou même de restituer aux centrales une partic du travail développé par la pesanteur dans les trains descendants et réduit ainsi dans une proportion très appréciable la dépense d'énergie nécessitée par l'exploitation des fortes rampes.

Si, sur les lignes de plaines, ces mêmes considérations ne peuvent plus guère être invoquées, la traction électrique y présente encore cependant dans bien des cas des avantages marqués.

Sur les réseaux suburbains, tout d'abord, l'exploitation électrique par rames réversibles à commande à unités multiples, qui permet seule un service réellement intensif, constitue un tel progrès que sa généralisation peut être envisagée dans un avenir relativement prochain.

Quant aux grandes lignes, la traction électrique peut également y être justifiéc sous certaines conditions.

Tout d'abord, au point de vue purement financicr, si les installations fixes que comporte l'équipement électrique des voies entraînent des dépenses de premier établissement très élevées et des frais d'entretien appréciables, ces charges ont pour contre-partie d'importantes économies d'exploitation : diminution du prix de l'énergie que les grandes centrales, soit hydrauliques, soit même thermiques, peuvent produire beaucoup plus économiquement qu'une locomotive, même en tenant compte des pertes dans le transport ; réduction des dépenses de graissage des machines en raison de la suppression de la consommation d'huile aux cylindres ; élimination des transports intérieurs de combustibles et des alimentations en eau ; économies sur le personnel des dépôts dont la suppression des manutentions de charbon et de déchets divers permet une réduction importante, et sur l'entretien du matériel moteur en raison de la disparition de la chaudière qui conslitue l'organe délicat des locomotives.

Cess diverses économies sont approximativement proportionnelles à l'intensité du trafic et peuvent, dès que celui-ci dépasse une certaine limite, rendre l'électrification avantageuse même sur une ligne à profil facile.

Mais, en dehors de ces considérations d'ordre financier, nous devons tenir compte de ce que la traction électrique constitue au point de vue du service des chemins de fer une amélioration importante, et procure des possibilités qu'il scrait vain d'envisager avec la traction à vapeur.

L'allure de la locomotive électrique étant moins sensible que celle de la machine à vapeur aux variations du profil, et son démarrage étant plus rapide, l'électrification permet de réaliser le relèvement de la vitesse moyenne de marche. D'autre part, la locomotive électrique étant dispensée des arrêts périodiques pour le remplissage du tender et le nettoyage du feu, les trajets peuvent être rendus plus directs. De la combinaison de ces deux effets résulte en faveur de la traction électrique une augmentation notable de la vitesse commerciale des trains.

Dans un autre ordre d'idées, les frais de traction se trouvant beaucoup diminués, il est possible, en exploitation électrique, d'augmenter sans frais excessifs le nombre des trains et de réaliser ainsi dans le service des améliorations considérables.

La suppression de la fumée est un avantage appréciable non seulement pour les riverains du chemin de fer mais aussi pour les voyageurs, particulièrement sur les lignes touristiques.

La traction électrique fait disparaître également tout danger d'incerıdic par les escarbilles et les flammèches de la locomotive. C'est là une considération importante sur les lignes qui traversent des régions boisées et tout particulièrement sur celle de Bordeaux à Hendaye où, à la traversée des forêts de pins des Landes, la Compagnie du Midi est actuellement obligée de ce fait à des mesures de surveillance et de défense extrêmement minutieuses.

Il est enfin une considération d'ordre général qui peut apporter en faveur de l'extension de la traction électrique un argument de grand poids. Etant donné que ce mode de traction implique l'établissement, le long de la plupart des voies ferrées, de lignes de transport d'énergie électrique, il est tout indiqué d'utiliser ces lignes à transporter non seulement le courant nécessaire au chemin de fer, mais aussi celui qui est destiné aux secteurs de distribution. Un grand progrès a été réalisé à ce point de vue par la décision prise par M. le Ministre des Travaux publics d'unifier désormais la forme du courant utilisé par les réseaux de chemin de fer et par les entreprises de transport d'énergie.

Ainsi les chemins de fer électrifiés étendront sur le territoire un réseau à mailles serrées de lignes électriques sur lequel les secteurs locaux pourront puiser leur énergie.

Les droits de péage qui seront perçus par le chemin de fer pour les transports d'énergie effectués sur les lignes permettront d'alléger dans une certaine mesure les lourdes charges qu'entraîne la construction de celles-ci et de transformer en opération profitable l'électrification de certaines artères qui, sans cela, ne pourrait être financièrement justifiée. Mais surtout le développcment qui en résultera dans l'utilisation industrielle et domestique de l'énergie électrique constituera pour les populations intéressées une source incalculable de bien-être et de progrès.

Prenons à titre d'exemple, l'électrification en cours sur le réseau des Chemins de fer du Midi.

En vue de la desserte de la ligne de Bordeaux à Hendaye au moyen des usines hydro-électriques de la vallée d'Ossau, la Compagnie construit en ce moment une puissante ligne de transport à 150.000 volts entre Laruns et Bordeaux. Cette ligne dont la capacité dépasse celle que nécessiteraient les seuls besoins 
du chemin de fer, sera, pour le surplus, mise à la disposition des autres producteurs d'électricité. Elle permettra, dès 1923, d'amener des Pyrénées pour desservir l'agglomération bordelaise toule la puissance que celle-ci est en mesure d'absorber.

Cette ligne comportera à Dax un poste intermédiaire qui pourra jouer le même rôle de centre de distribution pour la région des Landes.

De l'autre côté, une deuxième grande ligne à 150.000 volts, également en construction, reliera Lannemezan, point d'aboutissement de l'énergie électrique de la vallée d'Aure, à Toulouse. Ainsi grâce aux travaux de la Compagnie des Chemins de fer du Midi, les deux métropoles du Sud-Ouest seront à très bref délai mises à même d'augmenter considérablement leur consommation d'énergie électrique.

Mais ce n'est pas tout. Sur les lignes de transport à 150.000 volts la Compagnie du Midi branche des lignes à 60.000 volls qui suivront la voie ferrée pour l'alimentation des sous-stations de transformation produisant le courant de traction. Ces sousstations seront espacées de 20 à 30 kilomètres et placées en principe aux nœuds du chemin de far, c'est-à-dire, en général, dans les gares les plus importantes. Les lignes dont il s'agit auront, de leur côté, une capacité généralement supérieure aux bescins du chemin de fer, et pourront être utilisées prur d'autres transports. réalisation par la Compagnie des Chemins de fer du Midi. Nous nous limitons à la partie occidentale du réseau, où les travaux sont déjà en cours, c'est-à-dire aux lignes de Toulouse à Bayonne et de Bordeaux à Hendaye avec leurs embranchements et aux deux nouvelles traversées des Pyrénées partant de Toulouse par Ax-les-Thermes et de Pau par Oloron. Le développement des lignes à électrifier dans cette zone atteinl 1.782 kilomètres dont 545 en double voie.

Usines hydro-électriques. - Le courant destiné à alimenter ces lignes scra produit dans 8 usines hydro-électriques qui sont:

Les 2 usines de Soulom et d'Eget, déjà en service, équipées respeclivement pour des puissances de 21.000 et 35.000 chevaux ;

Les 3 usines échelonnées de la vallée d'Ossau, Artouste, Miegebat et le Hourat, actuellement en construction, et qui seront équipées au total pour 130.000 chevaux.

Enfin un autre groupe de trois usines échelonnées sur l'Ariège en amont d'Ax-les-Thermes qui ne sont encore qu'à l'état de projet et qui seront équipées pour 100.000 chevaux.

Le tableau ci-après donne pour chaque usine les hauteurs de chute, les débits maxima et moyens, la puissance et le mode d'équipement :

\begin{tabular}{|c|c|c|c|c|c|c|c|}
\hline \multirow{3}{*}{ Usine de Soulom. } & $\begin{array}{c}\text { IIAUTECR } \\
\text { de chute netle } \\
\text { en mètres }\end{array}$ & $\begin{array}{c}\text { DLEBIT } \\
\text { moyen en litres } \\
\text { par seconde }\end{array}$ & $\begin{array}{c}\text { DÉBIT } \\
\text { maximum }\end{array}$ & $\begin{array}{l}\text { PUISSANCE } \\
\text { '́quipée } \\
\text { en IIP }\end{array}$ & \multicolumn{2}{|c|}{ MODE D'EQUUIPEMENT } & $\begin{array}{l}\text { TYPE } \\
\text { des turbines }\end{array}$ \\
\hline & & & & & & & \\
\hline & 240 & 1.500 & 4.037 & 10.500 & \multirow{2}{*}{\multicolumn{2}{|c|}{$\begin{array}{l}3 \text { groupes de } 3.500 \mathrm{HP} \\
3-\mathrm{de} 3.500-\end{array}$}} & \multirow{2}{*}{$\begin{array}{l}\text { Pelton } \\
\text { Francis }\end{array}$} \\
\hline Basse chute......... & 106 & 4.400 & 9.225 & 10.500 & & & \\
\hline Usine d'Eget............ & 710 & 1.750 & 4.760 & 35.000 & - & de $5.000-$ & Pelton \\
\hline \multicolumn{8}{|l|}{ Usines de la vallée d'Ossau. } \\
\hline Artouste. $\ldots \ldots \ldots \ldots \ldots$ & 773 & 2.240 & 3.245 & 30.000 & - & de $10.000-$ & \multirow{3}{*}{$\begin{array}{l}\text { Pelton } \\
\text { Pelton } \\
\text { Francis }\end{array}$} \\
\hline Miegebat.............. & 380 & 5.400 & 10.600 & 50.000 & - & de $10.000-$ & \\
\hline Hourat............. & 204 & 8.100 & 17.200 & 50.000 & - & de $10.000 \ldots$ & \\
\hline \multicolumn{7}{|l|}{ Usines de l'Ariège: } & \\
\hline Saillens................ & 695 & 2.000 & 3.600 & & - & de $10.000-$ & \\
\hline Merens.............. & 188 & 4.000 & 10.500 & 100.000 & - & de $10.000-$ & \\
\hline Ax-les-Thermes..$\ldots \ldots \ldots$ & 330 & 6.820 & 13.700 & & - & de $10.000-$ & \\
\hline
\end{tabular}

Ainsi la traction électrique aboutira à ce résultat que, presque toutes les localités de quelque importance situées le long de la voie ferrée seront dotées d'un poste à 60.000 volts où les secteurs de distribution pourront venir s'alimenter.

On conçoit sans peine le développement qu'une pareille organisation pourra donner à l'activité industrielle et agricole des régions ainsi desservies.

Envisagée sous cet angle, la question de l'électrification des voies ferrées s'élargit singulièrement. Débordant le cadre de la technique des transports, elle se lie au problème de la diffusion de l'énergie électrique sur toute l'étendue du territoire et revêt ainsi une importance nouvelle et considérable, non seulement en ce qui concerne les intérêts régionaux, mais même au point de vue de l'intérêt national.

Comme suite à cet exposé général, nous donnons ci-après une brève description des travaux d'électrification en voie de
Toutes ces usines fourniront à leurs barres haute tension du courant triphasé 50 périodes 60.000 volts qui sera déversé sur les lignes de distribution du chemin de fer. En outre, une partie du courant provenant des usines de l'Ossau, d'Eget et de l'Ariège sera, en vue du transport à grande distance, transformé à 150.000 volts dans les postes élévateurs du Hourat, de Lannemezan et d'Ax-les-Thermes.

Lignes de transport à 150.000 volts. —L'énergie sera transportée au moyen :

$1^{\circ}$ D'une ligne double de 249 kilomètres de longueur de Laruns à Bordeaux par Dax, alimentée à Laruns par le poste élévateur du Hourat.

$2^{\circ}$ D'une ligne simple de 113 kilomètres de Lannemezan à Toulouse alimentée à Lannemezan par le courant venant de l'usine d'Eget.

$3^{\circ}$ Par une ligne double de 110 kilomètres d'Ax-les-Thermes à Toulouse alimentée par les usines de l'Ariège.

Enfin la mise en parallèle des différents groupements sera assurée par une ligne simple de 71 kilomètres reliant Pau à Lannemezan. 
Le tableau ci-dessous donne la puissance de transport de chaque tronçon :

$\begin{array}{cc}\begin{array}{c}\text { Longueur } \\ \text { en km, }\end{array} & \begin{array}{c}\text { Puissance } \\ -\end{array} \\ \text { de transport en kw. } \\ 37 & - \\ 72 & 100.000 \\ 140 & 100.000 \\ 71 & 70.000 \\ 113 & 50.000 \\ 110 & 30.000 \\ & 100.000\end{array}$

Chaque ligne à 50.000 volts est conslituée par 3 câbles de cuivre de 143 millimètres carrés de section. Un fil de terre en acier de 60 millimètres carrés de section protège la ligne contre les décharges atmosphériques. Le point le plus bas du fil inférieur est à 8 mètres du sol.

Les fils sont supportés par des chaînes de 9 isolateurs en porcelaine, soit du type assiette avec tige et capot scellés au ciment, soit du type Jeffery Dewitt à scellement au plomb. Le tout est soutenu par des pylônes métalliques en treillis d'environ 20 mètres de hauteur avec portées normales de 200 mètres.

La construction de ces pylônes a fait l'objet d'une étude particulière en vue d'abtenir autant que possible l'égalité de résistance depuis la base jusqu'au sommet et de réduire ainsi le poids du métal. On a adopté une construction consistant à attacher aux mêmes points des montants les croisillons de deux faces adjacentes du pylône, de manière à constituer le cloisonnement par une série de tétraèdres.

La base du pylône forme un carré de $1 \mathrm{~m} .50$ de côté.

En vue de faciliter le transport, les pylônes sont constitués par trois tronçons qui sont assemblés au moyen de boulons au moment du montage.

Le tronçon supérieur ainsi que les bras qui soutiennent les conducteurs, sont galvanisés par le procédé Schoop.

Dans les sections où la ligne est double, elle est constituée par deux lignes entièrement indépendantes.

Postes de transformation $150.000 / 60.000$ volts. - La tension du courant sera, comme on l'a vu, élevée de 60.00 à 150.000 volts dans les postes du Hourat, de Lannemezan et d'Ax-les-Thermes. Elle sera inversement abaissée de 150.000 à 60.000 volts dans les postes récepteurs de Pessae (près Bordeaux), Dax et PortetSaint-Simon (près Toulouse) et le courant ainsi abaissé sera déversé dans le réseau d'alimentation à 60.000 volts qui longe toutes les voies électrifiées.

Ces postes sont du type en plein air.

Leurs transformateurs statiques sont reliés respectivement aux barres à 150.000 volts et à 60.000 volts par des disjoncteurs à huile.

Les barres à 150.000 volts et les barres à 60.000 volls sont doubles et peuvent être réunies entre elles par des interrupteurs dans l'huile. Enfin les arrivées ct les départs des lignes se font également par l'intermédiaire de disjoncteurs.

Tous ces disjoncteurs à huile, encadrés de deux sélectionncurs, sont manœuvrés au moyen de relais permetlant de mettre hors circuit un appareil ou une ligne quelconque.

Les postes de Bordeaux, Portet-Saint-Simon et Dax sont dotés en outre de moteurs synchrones agissant comme compen- sateurs de phase et assurant la régularisation de la tension aux points d'arrivée.

Le tableau ci-dessous donne la constitution des divers postes à 150.000 volls :

\begin{tabular}{|c|c|c|c|}
\hline & TRANSFORMATEURS & $\begin{array}{l}\text { COMPENSATEURS } \\
\text { SYNCImONES }\end{array}$ & $\begin{array}{l}\text { PUISSANCE } \\
\text { TOTALE }\end{array}$ \\
\hline Pessac......... & $\begin{array}{c}7 \text { tranformateurs } \\
\text { de } 6.660 \mathrm{kw} \text { (dont } \\
1 \text { de rechange) }\end{array}$ & $2 \underset{15}{\text { groupes de }}$ & $\begin{array}{l}\text { kilowatts } \\
40.000\end{array}$ \\
\hline 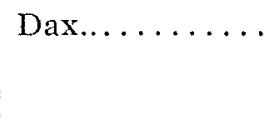 & $\begin{array}{l}4 \text { tranformateurs } \\
\text { de } 6.660 \mathrm{kw} \text { (dont } \\
1 \text { de rechange). }\end{array}$ & 2 groupes de & $20.00_{0}$ \\
\hline Hourat............ & $\begin{array}{l}7 \text { transformateurs } \\
\text { de } 6.660 \mathrm{kw} \text { (dont } \\
1 \text { de rechange). }\end{array}$ & & 40.000 \\
\hline Lannemezan...... & $\begin{array}{l}4 \text { transformateurs } \\
\text { de } 6.660 \mathrm{kw} \text { (dont } \\
1 \text { de rechange). }\end{array}$ & & 20.000 \\
\hline Portet-St-Simon. & $\begin{array}{l}4 \text { transformateurs } \\
\text { de } 6.660 \mathrm{kw} \text { (dont } \\
1 \text { de rechange. }\end{array}$ & $\begin{array}{l}2 \text { groupes de } \\
8.000 \mathrm{kw} .\end{array}$ & 20.000 \\
\hline Ax-les-Thermes & A l'étude. & & A l'étude. \\
\hline
\end{tabular}

Réseau de distribution à 60.000 volts. - L'énergie nécessaire au chemịn de fer est distribuéc au moyen d'un réscau à 60.000 volts qui suit en principe les lignes électrifiées sur toul leur parcours.

La ligne à 60.000 volts est généralement montée sur les poleaux qui supportent la ligne de contact.

Elle est constituéc par 3 câbles de cuivre de 100 millimètres carrés de section.

Sous-stalions de traction. - Le courant triphasé est converti en courant continu à 1.500 volts pour la traction dans les sousstations placées à des distances variables avec le profil de la ligne et le trafic, de manière que la chute de tension en ligne ne dépasse pas en service normal $20 \%$.

En fait, la distance de deux sous-stations voisines varie entre 13 et 31 kilomètres.

Sur les premières lignes équipées, la plupart des sous-stalions sont constituées avec des groupes de deux commutatrices à 750 volts en série. Cependant, à titre d'essaị, quelques sousstations ont été munies soit de commulatrices à 1.500 volts directs, soil de redresseurs à vapeup de mercure à 1.500 volts et à 12 pôles.

Le résultat de ces essais permettra de déterminer le type d'armement à adopter définitivement.

Les sous-stalions ont été construites suivanl un type spécialement éludié et particulièrement économique dans lequel tout l'armement à haute tension est placé à l'extérieur.

Le tableau ci-après indique les caractérisliques des sousstations de la section de Dax-Toulouse et de.ses embranchements. 


\begin{tabular}{|c|c|c|}
\hline $\begin{array}{c}\text { NOMS DES } \\
\text { SOUS-STATIONS }\end{array}$ & TYPE DES CONVERTISSEURS & $\begin{array}{l}\text { NOMBRE ET } \\
\text { PUISSANGE UITTARE } \\
\text { DES GROUPES }\end{array}$ \\
\hline 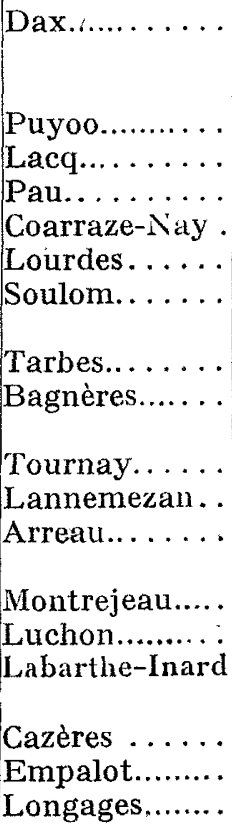 & 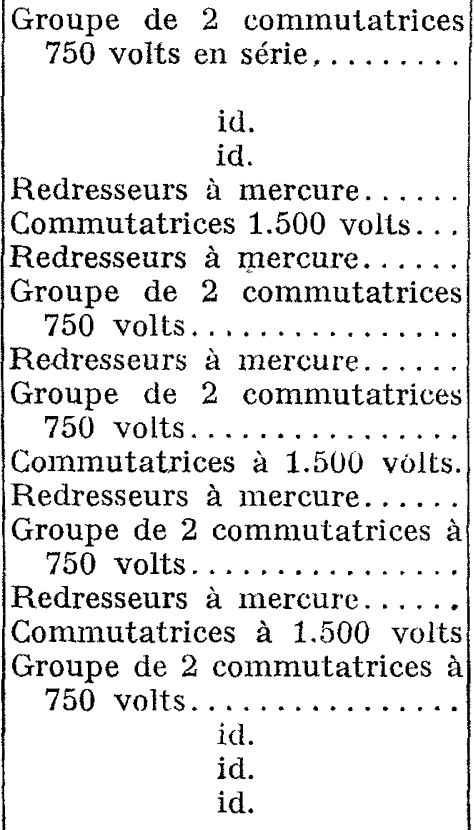 & $\begin{array}{l}2 \text { groupes de } \\
1.500 \mathrm{kw} \\
3 \times 1.500 \mathrm{kw} \\
1 \times 1.500 \mathrm{kw} \\
3 \times 1.200 \mathrm{kw} \\
3 \times 750 \mathrm{kw} \\
3 \times 1.200 \mathrm{kw} \\
\\
1 \times 1.000 \mathrm{kw} \\
3 \times 1.200 \mathrm{kw} \\
\\
1 \times 1.000 \mathrm{kw} \\
4 \times 750 \mathrm{kw} \\
4 \times 1.200 \mathrm{kw} \\
\\
1 \times 1.000 \mathrm{kw} \\
3 \times 1.200 \mathrm{kw} \\
2 \times 1.000 \mathrm{kw} \\
1 \times 1.500 \mathrm{kw} \\
2 \times 1.500 \mathrm{kw} \\
4 \times 1.500 \mathrm{kw} \\
1 \times 1.500 \mathrm{kw}\end{array}$ \\
\hline
\end{tabular}

Lignes de prise de courant. - La captation du courant se fait sur une ligne aérienne du type à double caténaire.

Cette ligne est constituée par ún câble porteur en acier de 80 millimètres carrés supportant tous les 9 mètres des pendules verticaux auxquels est suspendu un fil de cuivre de 100 millimètres carré dit porteur auxiliaire ; à ce dernier est suspendu lui-même, au moyen de petits pendules rigides très courts, le fil de travail en cuivre rainuré de 100 millimètres carré.

Les portées sont de 90 mètres en alignement et dans les courbes de rayon supérieur à 2.000 mètres, de 60 mètres dans les courbes de rayon compris entre 2.000 mètres et 800 mètres; enfin de 45 mètres dans les courbes de rayon inlérieur à 800 mètres.

Dans les courbeș, la ligne ne comporte pas d'antibalançant de sorte que, par suite de la tension du fil de travail, les pendules prennent une position inclinée vers l'intérieur de la courbe. Cette disposition permet à la ligne de contact de suivie presque rigoureusement l'axe de la voie et d'avoir une grande souplesse mème au voisinage des supports, conditions qui favorisent la captation du courant à grande vitesse. Elle a en outre l'avantage de compenser automatiquement dans une certaine mesure les variations de température, le fil de travail s'abaissant quand la température s'élève et se relevant quand la température s'abaisse.

Les tensions du fil de travail et du porteur auxiliaire sont réglées à 700 kilogrammes à la température de $15^{\circ}$ et sans vent.

En alignement, afin d'éviter sur la ligne les points durs, on a étudié un type spécial d'antibalançant élastique. Des essais sont en cours qui permettront même peut-être de supprimer entièremenl cet organe.

En vue de limiter l'étendue des accidents et de faciliter les recherches et les réparations, les caténaires sont divisées en sections alimentées séparément. Les points de coupure sont placés de part et d'autre de chaque gare, les voies de la gare, constituant elles-mêmes une ou plusieurs sections.

Les sectionnements en pleine voie sont à lames d'air ; les deux tronçons de la ligne chevauchent côte à côte sur une certaine distance de telle sorte que le pantographe passe de l'un à l'autre progressivement et sans choc.

En vue d'obtenir une captation de couranl aussi régulière que possible, les pantographes des locomoteurs sont munis de deux frotteurs suspendus élastiquement sur un balancier et comportant chacun deux lames de contacl en cuivre.

Le retour du courant se fait par les rails de roulement qui sont à cel effet réunis au moyen de connexions en cuivre soudées à l'arc électrique.

Les supports de la caténaire portent en même temps les feeders à courant continu, la ligne de distribution à 60.000 volts et, le long de certaines voies, une ligne triphasée à 10.000 volts servant à alimenter les gares en courant pour l'éclairage et la force motrice.

Protection du réseau. - La protection contre les surintensités et les surtensions du réseau a fait l'objet d'une étude particulièrement attentive.

Contre les surintensités, on a prévu sur les réseaux à 150.000 et à 60.000 volts un système de protection sélectif au moyen de disjoncteurs commandés par des relais à maximum, par des relais à retour de courant ou par des relais différentiels, disposés et réglés de telle manière qu'un court-circuit en ligne ait pour résultal la mise hors circuit automatique et immédiate de la partie avariée, sans affecter les postes ni les autres parties du réseau.

Les lignes à 150.000 volts sont protégées à leur entrée dans les sous-stations de traction par des disjoncteurs à maximum. D'autre part, sur le circuit de chaque commutatrice, un disjoncteur à action ultra-rapide pare aux effets d'un court-circuit survenant soit sur la ligne, soit à l'intérieur de la sous-station. Un relais empêche de coupler une commutatrice sur le réseau si la polarité n'est pas convenable, de même qu'un relais spécial à retour de courant installé sur le pôle négatif de chaque commutatrice, met celle-ci hors circuit si le courant vient à être inversé.

Enfin, un relais fait déclencher le disjoncteur à 60.000 volts si le courant triphasé vient à manquer, en vue d'éviter qu'au moment du rétablissement la commutalrice ne soit avariée.

Quant à la protection contre les surtensions, les lignes de transport à 150.000 volts sont tout d'abord munies d'un fil de terre destiné à les protéger contre les décharges atmosphériques. Les points neutres des circuits triphasés sont mis à la terre.

Les lignes à 150.000 volts ont été munies en outre de parafoudres électroly tiques. Dans les circuits à 60.000 volts on installe, en avant des appareils à protéger, des amortisseurs d'ondes consistant en combinaisons de bobines de self et de condensateurs au mica, et des déchargeurs composés d'une bobine de self à noyau de fer aboutissant à la terre.

Enfin, les appareils à 1.500 volts, commutatrices, redresseurs à mercure ou autres sont protégés par une bobine de self placée en série sur chaque départ à 1.500 volts et par une dérivation à la terre conslituée par un condensateur shunté par une résistance.

Protection des réseaux voisins. - Pour protéger les circuils voisins contre les effets d'induction, des rotations régulières ont été effectuées sur chaque ligne triphasée.

Pour les lignes à 150.000 volts on effectue un tour complet entre deux pôles consécutifs. Pour les lignes à 60.000 volts, on effectue un tiers de tour tous lés kilomètres.

Matériel moteur. - Le matériel moteur comporte à la fois des automotrices et des locomotives.

Les automotrices sont du type à 2 bogies moteurs ét à adhérence totale. Elles ont une puissance continue de 500 chevaux et une puissance unihoraire de $700^{\circ}$ chevaux. Elles peuvent atteindre la vitesse de 80 kilomètres à l'heure.

Une première série, obtenuc au moyen de la transformation 
des automotrices actiulles à courant monophasé, comprendra des voitures mixtes de tre ot ze classes à couloir latéral. Les nouvelles voitures seront de 3 e classe, à couloir central et à caisse entièrement métallique.

Les quatre moteurs de chaque automolrice, d'une puissance continue de 125 chevaux, sont suspendus par le nez.

Ils attaquent l'essieu au moyen d'une engrenage fixé non sur l'essieu mais sur le centre de la rone.

Les deux moleurs dun mème bogie sonl couplés en série of alimentés sous la tension totale de 1.500 volts, soit 750 volts par moteur. Ils sont à refroidissement naturel. La pleine puissance est oblemue a la vitesse de 45 kilometres I $^{\prime}$ heure el peut tre maintenue jusqu'a 55 kilometres a Theure, par lo shuntage des inducleurs.

Les automotrices sonl munies du controble à unités multiples.

Le mécanisme de contròle, du type série parallele, consiste un contacteurs actionnes par un arbre à cames.

Elles sont munies en outre d'un frein rhéostatique leur permettant dexercer un cffort de retenue dans les descentes.

Les locomolives destinées aux trains de marchandises et aux trains de vorageurs ommibus sont ègalement à deux bogies moleurs a adhence totale et armées de 4 moteurs suspendus par le nez. Leur puissance continue est de 1.000 chevaux et la puissance unihoraire de 1.400 chevaux. Les locomotives à vovageurs peuvent atteindre la vitesse de 80 kilomètres à l'heure, celles à marchandises la vilesse de 60 kilomètres. Ces machines ne different d'ailleurs entre elles que par le rapport d'engrenage.

Les deux bogies sont atteles entre eux et portent les appareils de traction el de tamponnement. Les efforls de traction et de choc sont ainsi transmis indépendamment de la caisse. Comme consęquence de cette disposition, le pivot d'un des bogies comporte un déplacement longitudinal.

Pour oblenir une langue période d'oscillation latérale de la aisst sur les bogies et diminuer ainsi rinkensite des chocs sur la voie dans les entrées en courbes, la surface d'appui des pivots est concave vers le sol, de telle sorte que la caisse oscille autour d'un axe longitudinal situé à peu près dans le plan des essieux, c'est-à-dire très bas. La stabilité de la caisse est assurée au moyen de 4 équilibreurs latéraux à ressorts réunissant la caisse aux châssis des bogies qui n'ont plus, par conséquent, de patins latéraux. Ces équilibres sont construits de manière à n'offrir aucune résistance à la rotation horizontale des bogies.

Chaque moteur transmet son effort à l'essieu correspondant au moyen de 2 roues dentées fixées sur les centres de roues.

Les moteurs sont alimentés directement à la tension de 1.500 volts. Leur puissance continue est de 250 chevaux et la puissance unihoraire de 350 chevaux. Ils sont ventilés artificiellement.

Les locomotrices à voyageurs peuvent réaliser leur puissance continue à la vitesse de 50 kilomètres à l'heure et la maintenir par le shuntage des inducteurs jusqu'à 74 kilomètres à l'heure. Pour les locomotives à marchandises, les chiffres ci-dessus sont respectivement de 30 et 45 kilomètres à l'heure.

Toutes ces machines sont munies du freinage, par récupération qui permet de relenir à la descente le train qu'elles penvent remorquer à la montée sur la même déclivité. Elles sont munies du contrôle série parallèle à contacteurs et arbre à cames et disposées pour la commande par unités multiples.

Les services auxiliaires sont assurés au moyen d'un groupe moteur-générateur de 72 kilowatts comprenant un moteur alimenté à 1.500 volts qui actionne d'une part une génératricè à 120 volts, d'autre part une génératrice à tension variable pour lexcitation des moleurs pendant la récupération et enfin les 2 ventilateurs des moteurs.

50 locomotives de ce type sont actuellement en construction et les premières d'entre elles entreront en service dans le courant de la présente amnée.

En dehors de ces machines, la Compagnie du Midi a mis à l'étude pour le service des trains express des loconiotives de grande vilesse d'un type entièrement nouveau pouvant atteindre 120 kilomètres à lheure. Deux exemplaires en ont élé mis en construction à titre d'essai et seront livrés dans quelques mois.

\title{
Services que l'Agriculture et la Houille Blanche peuvent attendre du Reboisement.
}

\author{
Par M. PAUL DESCOMBES, Ingênieur en chef honoraire des Manufactures de l'Etat.
}

L'agriculture rançaisè a beaucoup pâti de la sécheresse en 1921. dans ses vécoltes comme dans son betail, et l'industrie de Is houille blanche a sourfert êgalement de l'insuffisance des eaux. Cittle rarete des exux provient en grande partie de ce que les monlagnus swnt insulfisamment boisëes : et les enseignements de 1921. qui viennent confirmer cenx de la guerre, ne doivent pas pine perdus de vur.

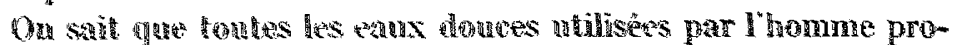
vinument de latmosphène, soil swus forme de pluies ou neiges mesurables par les plowionnetrn's, soil swus forme de condensations

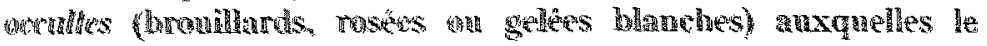
phoviometro ast insemsiblew.

1.

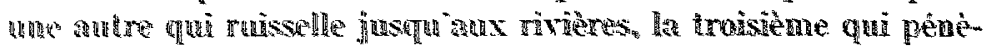

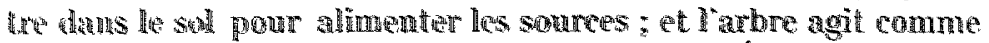
regulatever des eanx, en dimimuant lo ruissellemed pour aug-

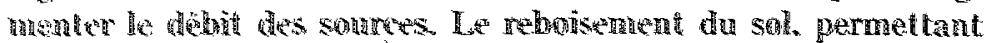

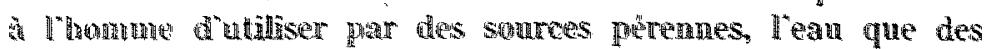

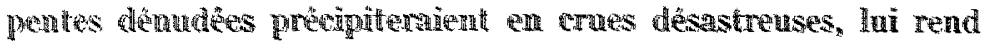

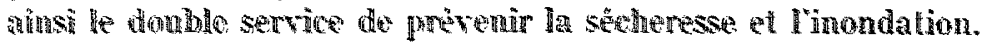

De son côté, l'eau du brouiliard et des rosées se dépose bien plus abondamment sur les arbres que sur le sol nu. Le reboisement, fournissant à l'homme un supplément d'eaux utilisables, lui donne de la sorte une seconde défense contre la sécheresse et lui permet de renforcer les débits d'étiage avec des eaux supplémentaires qui ne font courir aucun risque d'inondation,

\section{$*^{*} *$}

Il convient d'examiner ici dans quelle mesure le reboisement produit ce renforcement du débit.

La proportion de ce renforcement serait sans doute universellement connue depuis longtemps, si les expériences que I'Ingénieur en chef Conte-Grandchamps résumait dans son rapport du 10 janvier 1862 avaient été continuées: Nous avons fait, disait-il, dans le département de la Loire, pendant trois années consécutives, des expériences tendant à établir l'influence des forêts de sapins sur le débit des sources. Ces expériences nous ont démontré que, dans les terrains granitiques situés à 1.000 mètres environ au-dessus du niveau de la mer, le dẻbit des sources est deux fois plus considérable dans les terrains boisés que dans 